\title{
Electrical Properties of WSe 2 -x Single Crystals Grown By Direct Vapour Transport Technique
}

\author{
Rajiv Vaidya ${ }^{1}$, Mehul Dave ${ }^{2}$ and Kaushik R. Patel ${ }^{3 *}$ \\ ${ }^{1}$ Sheth P. T. Arts and Science College, Godhra, Dist. Panchmahal, Gujarat, India 389001 \\ ${ }^{2}$ N. V. Patel Pure and Applied Science College, Vallabh Vidyanagar, Gujarat, India 388120 \\ ${ }^{3}$ Biogas Research and Microbiology Department, Gujarat Vidyapith, Sadra, Gujarat, India \\ 382320 \\ Email: $\frac{1 \text { rajivdvaidya@gmail.com }}{{ }^{2} \text { addave1977@yahoo.com }}, \frac{{ }^{3} \text { krpatel@ gujaratvidyapith.org }}{{ }^{2} \text { orresponding Author }}$
}

\begin{abstract}
:
The single crystals of WSe $\mathrm{W}_{2 x}$ were grown by direct vapour transport technique. The grown crystals possess hexagonal crystal structure. The resistivities of the as grown crystals were measured using van der Pauw method. The Hall parameters of the grown crystals were determined at room temperature from Hall effect measurements. Electrical resistivity measurements were performed on this crystal in the temperature range 303-423 K. The crystals werefound to exhibit semiconducting nature in this range. The activation energy and anisotropy measurements were carried out for this crystal. The value of resistivity is increases with increasing selenium content. The results obtained are discussed in detail.
\end{abstract}

Key Words: Direct vapour transport technique, Single crystals, Four probe technique, Resistivity, Hall effect

\section{Introduction:}

Lamellar dichalcogenides of group-VI transition metal elements Mo and W are promising semiconducting materials for the photoelectrochemical (PEC) conversion of solar energy to electricity, because of their high absorption coefficients in the visible and near infrared [1] and their excellent output stability as photoelectrods in polyiodide electrolytes [2,3]. At large number of investigators [4] have therefore, focussed their attention on the PEC studies of $\mathrm{MoSe}_{2}$ and $\mathrm{WSe}_{2}$. However, very little work appears to have been done on offstoichiometric crystals of tungsten diselenide. Since these materials exhibit semiconducting behaviour, it is worth studying them from the electrical properties point of view. It is obvious that these properties are of primary importance in the evaluation of these semiconducting materials for device fabrication.

The quasi two dimensional structure of these crystals resemble a stacking of sandwiches in each of which a plane of hexagonally arrayed tungsten (W) atoms lies between two planes of similarly arrayed selenium (Se) atoms i.e. Se - W - Se. Within a layer, the bonds are strong, while between adjacent layers they are remarkably weak. As a consequence, the crystals possess facile basal cleavage, lubricity and marked anisotropy in their physical and chemical properties. 
Since $\mathrm{WSe}_{2-\mathrm{x}}$ possesses a semiconducting nature, it was decided to study their electrical properties. In this research paper attention has been paid to the resistivity and Hall effect measurements on single crystals of $\mathrm{WSe}_{2-\mathrm{x}}$ grown by a direct vapour transport technique. The layered compounds are indeed anisotropic and there is a great variation in the anisotropy ratio amongst them. Measurements of c-axis resistivity of $\mathrm{WSe}_{2-\mathrm{x}}$ in the temperature range $313 \mathrm{~K}-423 \mathrm{~K}$ have also been undertaken. The determination of anisotropy ratios and their variation in the temperature $313 \mathrm{~K}-423 \mathrm{~K}$ have been carried out and the results have been presented and discussed.

\section{Experiment}

\subsection{Resistivity along the Basal Plane by van der Pauw method:}

The room temperature resistivity perpendicular to c-axis (i.e. along the basal plane) was investigated for $\mathrm{WSe}_{2-\mathrm{x}}(\mathrm{x}=0.01,0.04,0.07$ and 0.10$)$ single crystals using the van der Pauw method. A graph exhibiting the variation of resistivity versus composition is given in Figure1. It is clear from this plot that resistivity shows a decreasing trend with respect to increasing value of $\mathrm{x}$.

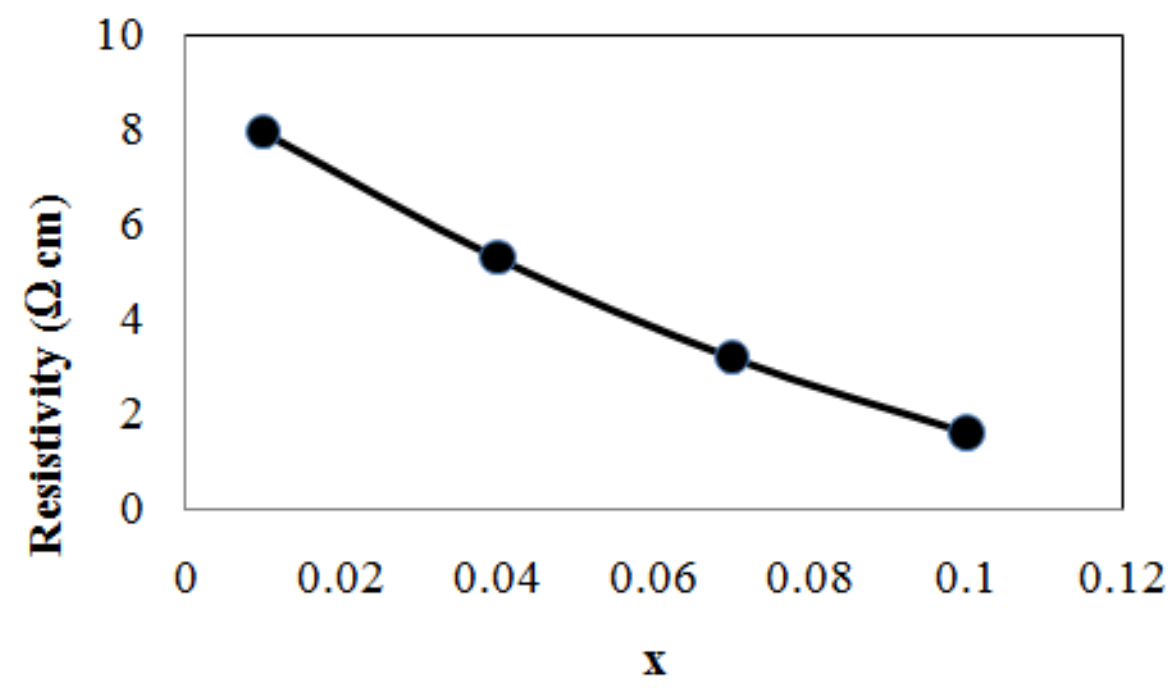

Figure-1: Variation of resistivity with composition $\mathrm{x}$ for $\mathrm{WSe}_{2-\mathrm{x}}$ single crystals

\subsection{Variation of Resistivity in the Low Temperature Range:}

The low temperature resistivity measurements of $\mathrm{WSe}_{2-\mathrm{x}}$ for $\mathrm{x}=0.01,0.04,0.07$ and 0.10 samples along the basal plane were done by the four probe set up developed by Scientific Solutions, Mumbai in the temperature range $153 \mathrm{~K}$ to $293 \mathrm{~K}$. Figure 2 shows the variation of resistivity with temperature for representative samples of $\mathrm{WSe}_{1.99}, \mathrm{WSe}_{1.96}$, $\mathrm{WSe}_{1.93}$ and $\mathrm{WSe}_{1.90}$ respectively. It is seen that in all cases the resistivity increases with decreasing temperature, thereby indicating that the samples show a classical semiconducting behaviour. The Plots shown in figure 2 give a match fit to the representative equation,

$\rho=\rho_{0} \exp \left(-\mathrm{E}_{\mathrm{a}} / \mathrm{k}_{\mathrm{B}} \mathrm{T}\right)$. 
From the slopes of the straight lines, the values of the activation energies for the different samples have been calculated using the formula, $\mathrm{E}_{\mathrm{a}}=2.303 \times \mathrm{k}$ x slope $\mathrm{eV}$

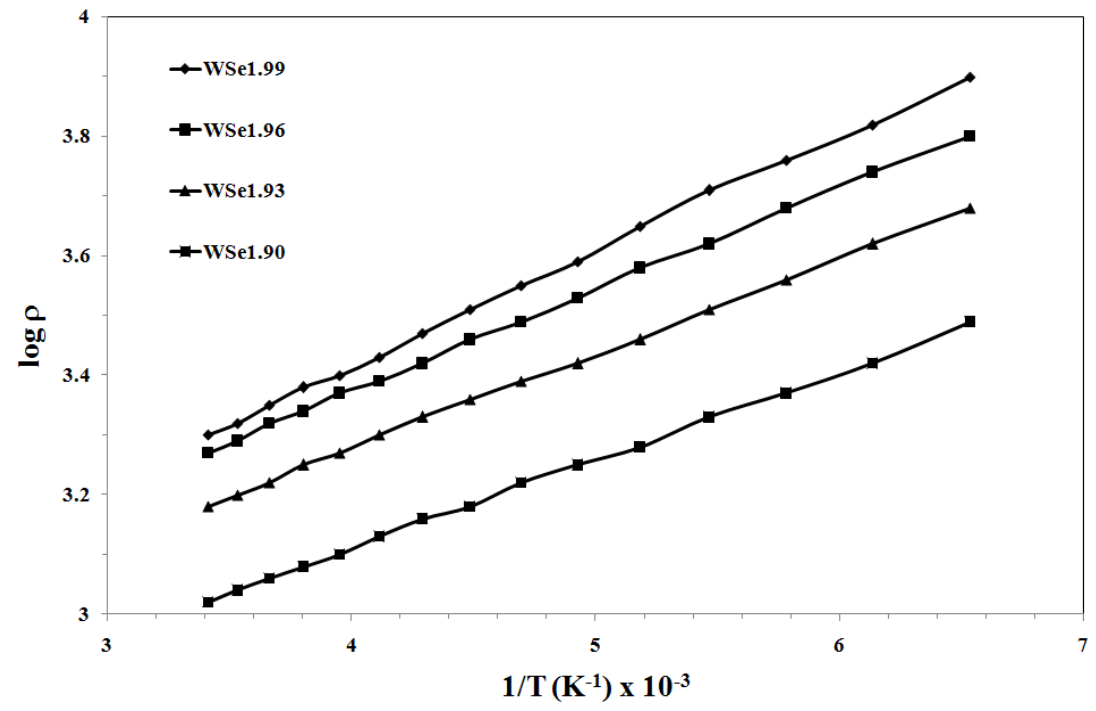

Figure-2: Variation of resistivity with inverse of temperature for $\mathrm{WSe}_{2-\mathrm{x}}$ single crystals

The values thus obtained are given in Table 1. The variation of $E_{a}$ with composition is represented in Figure 3. It is seen that activation energy decreases with increase in $x$.

Table-1: The activation energies for the different samples.

\begin{tabular}{|c|c|c|c|}
\hline $\mathbf{X}$ & Composition & Resistivity & Activation energy \\
\hline $\mathbf{0 . 0 1}$ & $\mathrm{WSe}_{1.99}$ & 7.94 & 0.038 \\
\hline $\mathbf{0 . 0 4}$ & $\mathrm{WSe}_{1.96}$ & 5.29 & 0.034 \\
\hline $\mathbf{0 . 0 7}$ & $\mathrm{WSe}_{1.93}$ & 3.18 & 0.031 \\
\hline $\mathbf{0 . 1}$ & $\mathrm{WSe}_{1.90}$ & 1.61 & 0.029 \\
\hline
\end{tabular}

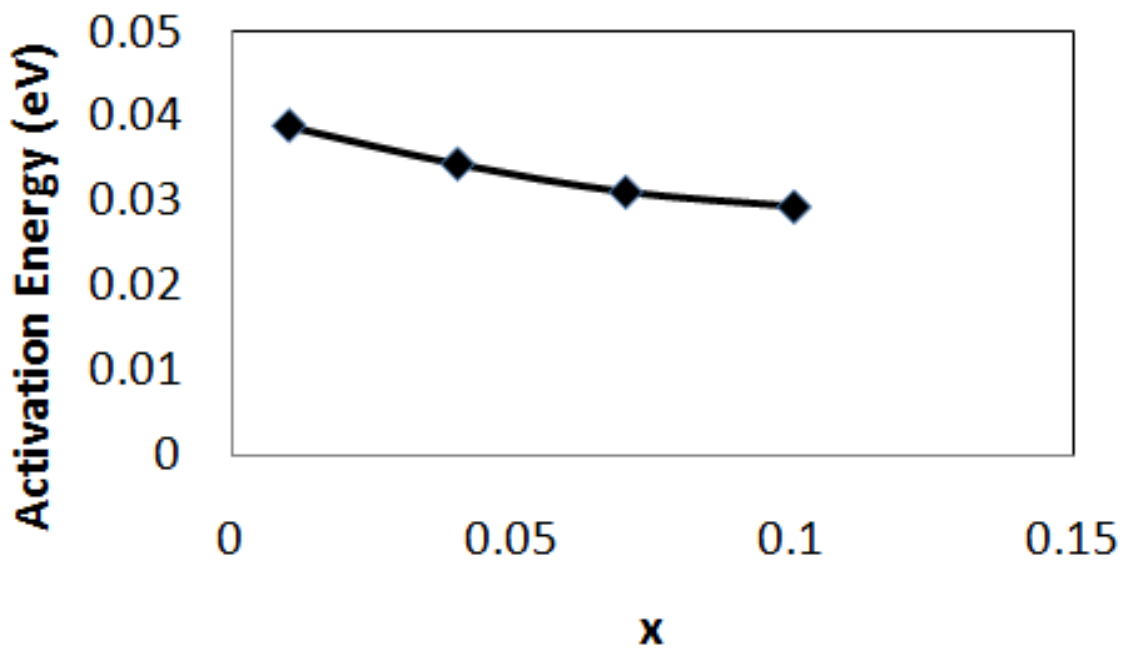

Figure-3:Variation of activation energy with composition. 


\subsection{High Temperature Resistivity Measurement Perpendicular to c-axis $\left(\rho_{\perp}\right)$ :}

The high temperature resistivity measurements perpendicular to c-axis i.e along the basal plane were carried out on single crystals of WSe2-x $(x=0.01,0.04,0.07$ and 0.10$)$ using the four probe technique. Figure 4shows the variation of resistivity with temperature, similar to resistivity measurements in the low temperature range. Here too, the resistivity decreases with increase in temperature. The activation energy of charge carriers for each samples was calculated using equation 2 and are represented in Table 2. The variation of activation energy with composition is shown in Figure 5. Here also, it is seen that similar to low temperature the activation energy decreases with increases in the value of $\mathrm{x}$.

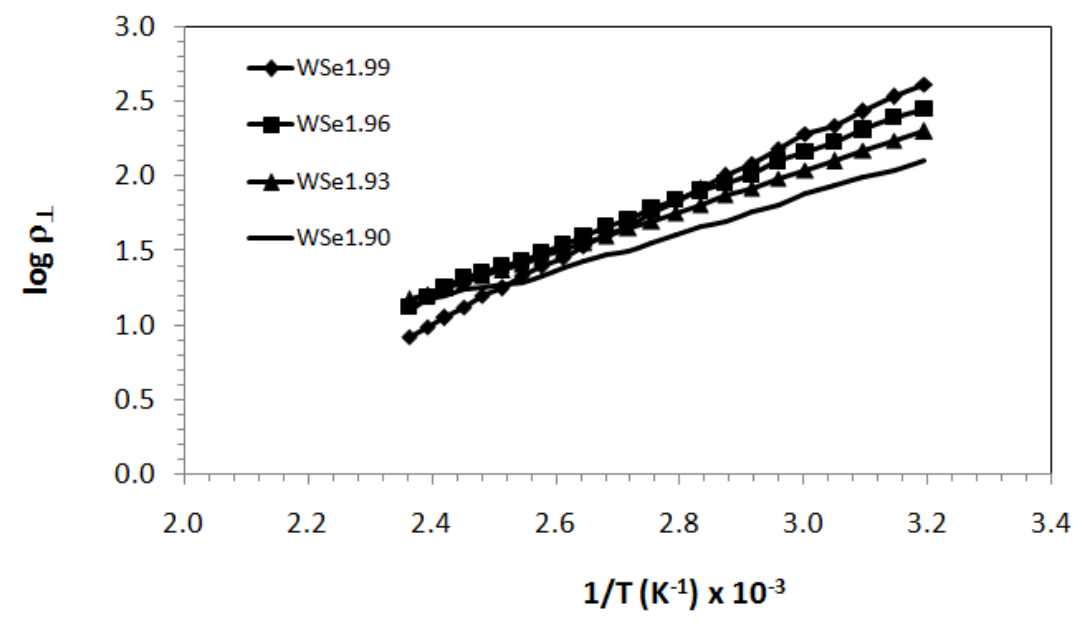

Figure-4: Variation of resistivity with inverse of temperature

Table-2: The activation energy of charge carriers for each samples.

\begin{tabular}{|c|c|c|}
\hline$x$ & Composition & $\begin{array}{c}\text { Activation } \\
\text { energy }\end{array}$ \\
\hline 0.01 & $\mathrm{WSe}_{1.99}$ & 0.41 \\
\hline 0.04 & $\mathrm{WSe}_{1.96}$ & 0.31 \\
\hline 0.07 & $\mathrm{WSe}_{1.93}$ & 0.27 \\
\hline 0.1 & $\mathrm{WSe}_{1.90}$ & 0.26 \\
\hline
\end{tabular}

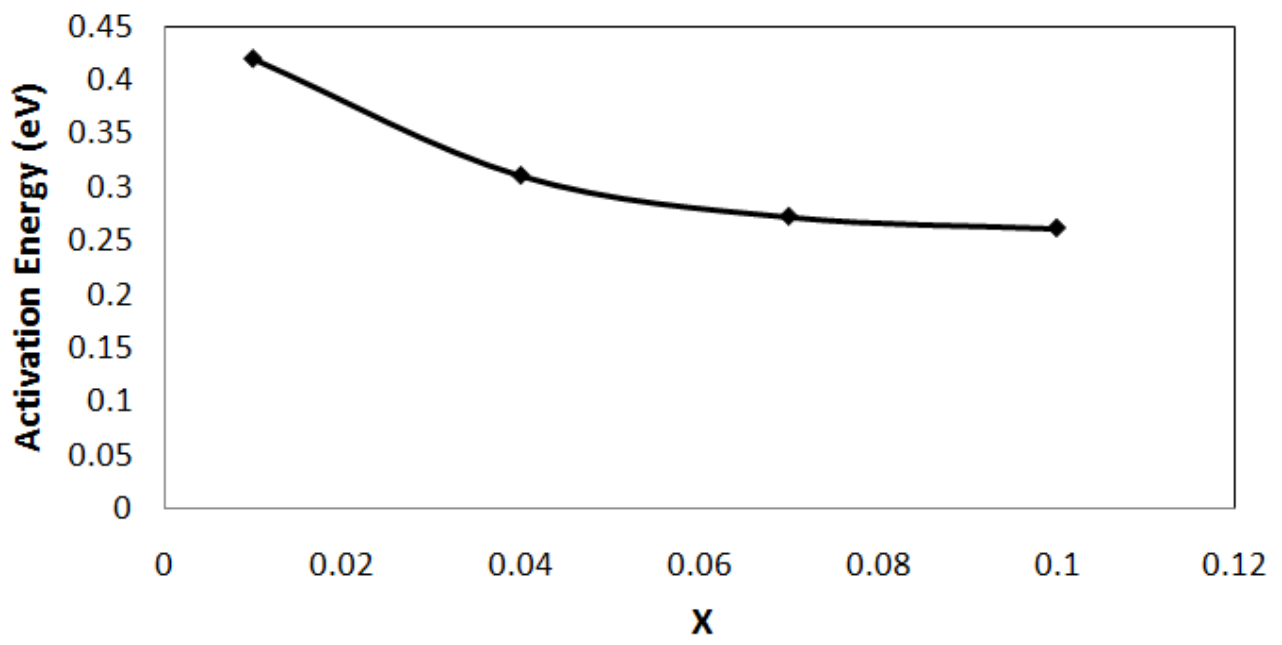

Figure-5: Variation of activation energy with composition. 


\subsection{High Temperature Resistivity Measurement Parallel to c-axis $\left(\rho_{\|}\right)$:}

For carrying out the electrical resistivity measurement parallel to c-axis i.e. normal to the basal plane, the same sample which were used for the resistivity measurement perpendicular to c-axis were used. Starting form room temperature, the temperature of the sample in each case was increased slowly in steps of $5 \mathrm{~K}$ until a temperature of $423 \mathrm{~K}$ was reached. At each temperature corresponding values of resistivity of representative samples were evaluated. The observed change of resistivity with temperature is shown in Figure 6. It is seen from this figure that resistivity in each case decreases with increase in temperature.

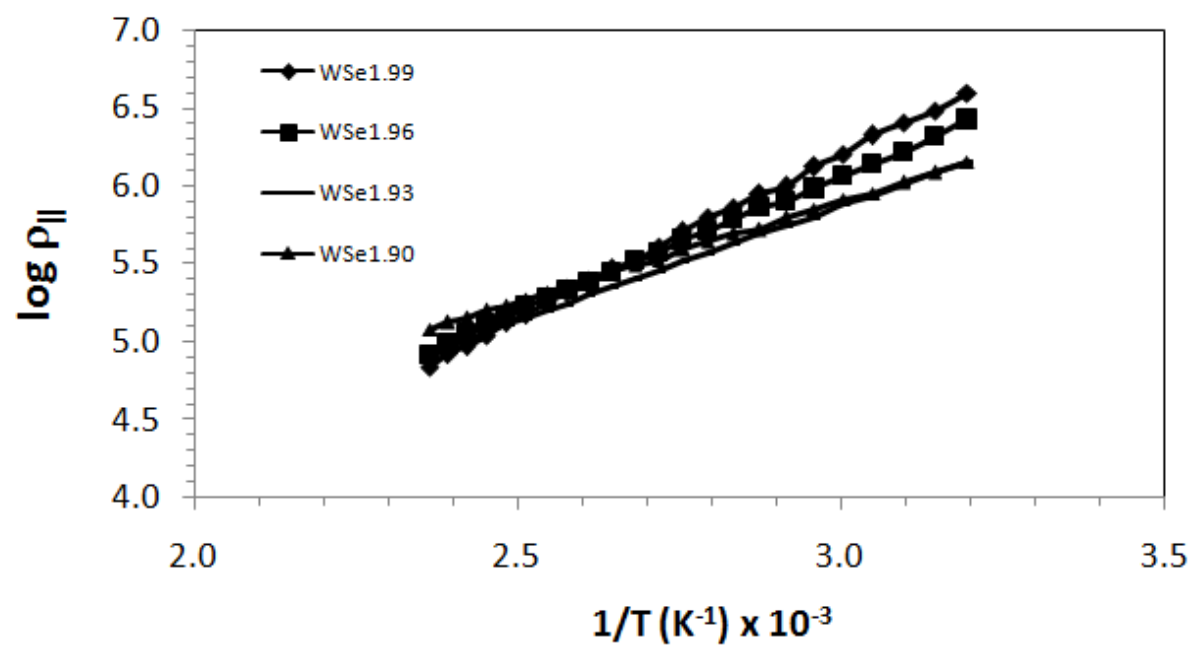

Figure-6: Variation of resistivity with inverse of temperature

From the slopes in Figure 6, the activation energies have been determined and the variation of activation energy with composition is given in Figure 7. A careful study shows that in comparison to resistivity along the basal plane, the resistivity is considerably high in a direction normal to the basal plane.

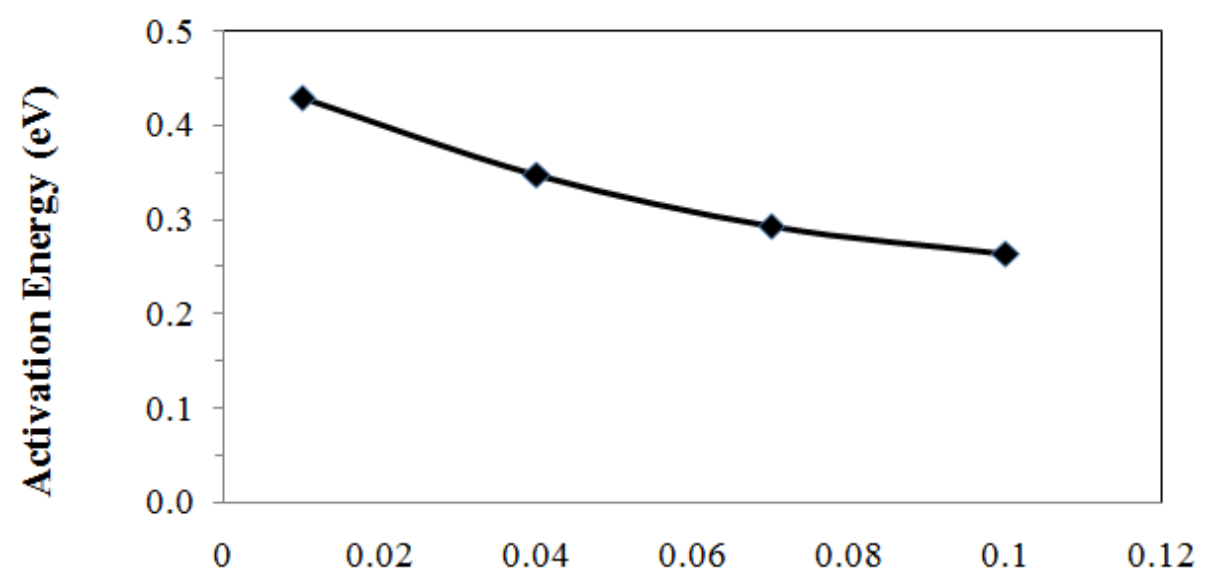

$\mathbf{x}$

Figure-7: Variation of activation energy with composition 


\subsection{Anisotropy Ratio $\left(\rho_{\|} / \rho_{\perp}\right)$ and its variation with Temperature:}

Using the resistivity values perpendicular to the c-axis and the resistivity values parallel to c-axis, the anisotropy ratio $\rho_{\|} / \rho_{\perp}$ for all the different samples has been determined in the temperature range $313 \mathrm{~K}$ to $423 \mathrm{~K}$. One important derivative, which emerges from the data, is that anisotropy ratio decreases with an increase in temperature shown in Figure 8. This trend is maintained by all non-stoichiometric samples of tungsten diselenide irrespective of their composition.

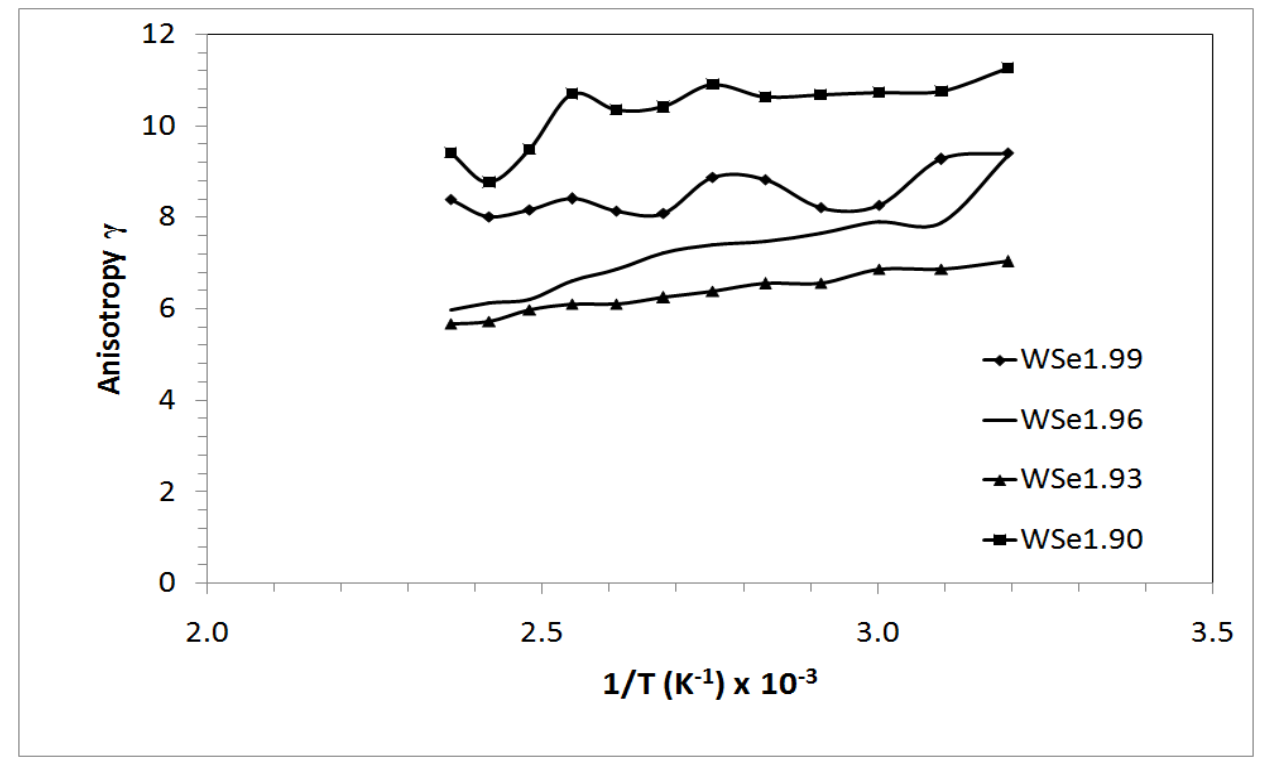

Figure-8: Variation of anisotropy with inverse of temperature

\subsection{Hall Effect Measurements:}

The modification of the van der Pauw technique has been used to evaluate the type, mobility and carrier concentration of $\mathrm{WSe}_{2-\mathrm{x}}(\mathrm{x}=0.01,0.04,0.07$ and 0.10$)$ single crystals. Hall mobility of the samples was determined by measuring the change in resistance $(\Delta R)$. When magnetic field was applied perpendicular to a sample, the Hall mobility $\left(\mu_{H}\right)$ is given by the relation,

$$
\mu_{\mathrm{H}}=(\mathrm{t} \Delta \mathrm{R}) / \mathrm{B} \rho \text {. }
$$

The results obtained from Hall effect measurements for representative samples in each case are given in Table 3. The range of magnetic field was varied from 0.25 to 10.35 KGauss. Knowing the values of t, B, R and $\rho$, the mobility of charge carriers was calculated using the above equation. The Hall coefficients and carrier concentrations were calculated using the formulae,

$$
\begin{aligned}
& \mathrm{R}_{\mathrm{H}}=\mu_{\mathrm{H}} \times \rho . \\
& \mathrm{p}=1 /\left(\mathrm{R}_{\mathrm{H}} \mathrm{e}\right) .
\end{aligned}
$$

The positive values of Hall coefficients clearly confirm that majority charge carriers in $\mathrm{WSe}_{2-\mathrm{x}}$ single crystals are holes. 
Table-3: The Hall parameters.

\begin{tabular}{|c|c|c|c|c|}
\hline $\mathbf{x}$ & Composition & $\begin{array}{c}\text { Hall co- } \\
\text { efficient }\end{array}$ & Mobility & $\begin{array}{c}\text { Carrier } \\
\text { Concentration }\end{array}$ \\
\hline $\mathbf{0 . 0 1}$ & $\mathrm{WSe}_{1.99}$ & 792.3 & 363.9 & $1.25 \times 10^{16}$ \\
\hline $\mathbf{0 . 0 4}$ & $\mathrm{WSe}_{1.96}$ & 539.6 & 490.5 & $8.81 \times 10^{16}$ \\
\hline $\mathbf{0 . 0 7}$ & $\mathrm{WSe}_{1.93}$ & 419.2 & 520.9 & $2.82 \times 10^{17}$ \\
\hline $\mathbf{0 . 1}$ & $\mathrm{WSe}_{1.90}$ & 292.3 & 531.8 & $8.16 \times 10^{17}$ \\
\hline
\end{tabular}

\section{Results \&Discussion:}

From the variation of electrical resistivity with temperature for all the compositions of $\mathrm{WSe}_{2-\mathrm{x}}$, it can be seen that with increase in temperature there is a gradual decrease in resistivity for all values of $\mathrm{x}$. So it implies that the temperature coefficient of resistance $\alpha$ is less than zero. This indicates that all the members of the series $\mathrm{WSe}_{2-\mathrm{x}}(\mathrm{x}=0.01,0.04,0.07$ and 0.10 ) possess a semiconducting nature.

Further, the room temperature values of $R_{H}$ obtained from d.c. Hall effect measurement on all the non-stoichiometric $\mathrm{WSe}_{2-\mathrm{x}}$ crystals used in the resistivity measurements described above are positive. This clearly shows that all of them exhibit a ptype semiconducting behaviour. The p-type character of $\mathrm{We}_{2-\mathrm{x}}$ is similar to that observed by Bonnet et al [5] for $\mathrm{MoTe}_{2-\mathrm{x}}$. This p-type character of $\mathrm{WSe}_{2-\mathrm{x}}$ can be assumed to be related to the lacunar sites which appear spontaneously in a crystal grown without any transporting agent and the concentration of which is a function of the temperature during the crystal growth. It is well known that these lacunar sites introduce accepter levels in the forbidden gap [6]. The presence of such acceptor level in $\mathrm{WSe}_{2-\mathrm{x}}$ is confirmed from the determination of the activation energies for all the members of the series in the low temperature range from $153 \mathrm{~K}$ to $293 \mathrm{~K}$.

From the values of activation energies, it can be noticed that $E_{a}$ decreases with increasing $\mathrm{x}$ in $\mathrm{WSe}_{2-\mathrm{x}}$. Moreover, it is seen that resistivity decreases as $\mathrm{x}$ increases, so it is quite reasonable to assume that with increasing value of $x$, the activation energy $E_{a}$ should also show a decreasing trend.

From the Hall parameters, it is seen that the concentration of the acceptor sites increases as the selenium content decreases in the compound. The decrease in resistivity and increase in mobility of the charge carriers is consistent with this behaviour.

The random potential of the acceptor charge centres induces a broadening of the corresponding acceptor levels into narrow bands. As a result the acceptor broadened level goes closer to the valence band and the acceptor level goes on decreasing with the increase in the non-stoichiometry. This is indeed true and can be seen from the decrease in the value of activation energy with increase in $x$ in all samples under investigation. The trend of decrease in activation energy with increasing $x$ observed in low temperature range is maintained even upon extending the range of resistivity measurement to $313 \mathrm{~K}$ to $423 \mathrm{~K}$. 
It is seen from the experiments on high temperature resistivity measurement parallel to c-axis that compared to the resistivity along the basal plane, the resistivity is about $10^{3}$ times higher. Further, it is seen from the values of anisotropy ratios that it decreases with increase in temperature which means anisotropic nature of the samples decreases with raise in temperature. This is possible because an increase in temperature may lead to production of vacancies which give rise to an increase in the value of resistivity thereby leading to a decrease in the value of the anisotropy ratio.

\section{Conclusions:}

$>$ The resistivity, both normal as well as along the basal plane decreases with increase in the temperature, which indicates the semiconducting nature of the grown crystals.

$>$ The electrical resistivity decreases with increasing the selenium proportion in the $\mathrm{WSe}_{2-\mathrm{x}}$ series. Their variation with composition $\mathrm{x}$ in $\mathrm{WSe}_{2-\mathrm{x}}$ is consistent with decrease in resistivity with increase in $\mathrm{x}$.

$>$ The measurements of resistivity parallel to c-axis have clearly revealed the anisotropic nature of the $\mathrm{WSe}_{2-\mathrm{x}} \mathrm{samples}$. Further, it has been shown that conduction process along the c-axis is nearly the same as that along the basal plane except the strong anisotropy.

> Activation energy obtained from the Figure 2 and Figure 4. It indicates that measured resistivity is the results of the extrinsic semiconducting nature of the crystals.

$>$ In all the cases, the resistivity $\left(\rho_{\|}\right)$parallel to c-axis is higher than the resistivity $\left(\rho_{\perp}\right)$ perpendicular to c-axis.

$>$ A schematic band structure proposed by Wilson and Yoffe has been suitably modified to explain the resistivity and Hall effect measurement in $\mathrm{WSe}_{2-\mathrm{x}}$ single crystals The positive value of Hall coefficient clearly indicates that all grown crystals are p-type in nature.

\section{References:}

\subsection{Journal Article}

[1] C. D. Scott and R. E. Smalley, "Diagnostic Ultrasound: Principles and Instruments", Journal of Nanosci. Nanotechnology. vol. 3, no. 2,(2003), pp. 75-80.

\subsection{Book}

[2] H. S. Nalwa, Editor, "Magnetic Nanostructures", American Scientific Publishers, Los Angeles, (2003).

[1] F. Frindt and A. D. Yoffe 1963, "Physical properties of layer structures: optical properties and photoconductivity of thin crystals of molybdenum disulphide" Proceeding Royal Society, Vol. No. 273 (1963) pp. 69.

[2] H. Tributsch and Ber Bunsenges, Phys. Chem, Vol. No.81 (1977) pp361.

[3] H. Tributsch, Strut. Bonding, Vol. No.49 (1982) pp. 127.

[4] A. Aruchamy, Ed. Photoelectrochemistry and Photovoltaics of Layered Semiconductors (Kluwer Academic, Dordrecht) (1992)

[5] A.Bonnet, A.Conan, M.Spiesser and M.Zoater, J.Phys., (France), Vol. No.49(1988) 803.

[6] A. Conan, A. Bonnet, A. Amrouche and M. Spiesser, "Semiconducting properties and band structure of $\mathrm{MoTe}_{2}$ single crystals” J. Physique, Vol. No. 45 (1984) pp. 459-465. 